\title{
Validação do balanço de radiação obtido a partir de dados MODIS/TERRA na Amazônia com medidas de superfície do LBA
}

\begin{abstract}
Gabriel de OLIVEIRA ${ }^{1}$, Elisabete Caria MORAES ${ }^{1}$
RESUMO

Este estudo tem como objetivo estimar os componentes do balanço de radiação em duas regiōes do estado de Rondônia (sudoeste da Amazônia brasileira), a partir de dados do Moderate Resolution Imaging Spectroradiometer (MODIS/TERRA) por intermédio do modelo Surface Energy Balance Algorithms for Land (SEBAL), e validar os resultados com informaçôes adquiridas por torres micrometeorológicas do projeto LBA sob as condiçóes de pastagem (Fazenda Nossa Senhora Aparecida) e floresta (Reserva Biológica do Jaru). A implementaçáo do modelo SEBAL foi realizada diretamente sobre os dados MODIS e incluiu etapas envolvendo o cômputo de índices de vegetação, albedo e transmitância atmosférica. A comparação das estimativas geradas a partir de dados MODIS com as observaçôes resultou em erros relativos para a condição de pastagem variando entre 0,2 e 19,2\%, e para a condição de floresta variando entre 0,8 e 15,6\%. A integração de dados em diferentes escalas constituiu uma proposição útil para a estimativa e espacialização dos fluxos de radiação na região amazônica, o que pode contribuir para a melhor compreensão da interaçáo entre a floresta tropical e a atmosfera e gerar informaçóes de entrada necessárias aos modelos de superfície acoplados aos modelos de circulação geral da atmosfera.
\end{abstract}

PALAVRAS-CHAVE: balanço de radiação na superfície, albedo, sensoriamento remoto, modelo SEBAL, radiômetros.

\section{Validation of net radiation obtained from MODIS/TERRA data in Amazonia with LBA surface measurements}

\section{ABSTRACT}

This study aims to estimate the components of net radiation in two regions located in the state of Rondônia (southwest of the Brazilian Amazon), using Moderate Resolution Imaging Spectroradiometer (MODIS/TERRA) data based on Surface Energy Balance Algorithms for Land (SEBAL) model, and to validate the results with information acquired by the micrometeorological towers of LBA under the conditions of pasture (Fazenda Nossa Senhora Aparecida) and forest (Reserva Biológica do Jaru). Implementation of SEBAL model was performed directly on the MODIS data and included steps involving the computation of vegetation indices, albedo and atmospheric transmittance. Comparison between estimates from MODIS data and the observations showed relative errors for the condition of pasture between 0.2 and $19.2 \%$, and for the condition of forest ranging between 0.8 and $15.6 \%$. The integration of data at different scales was a useful proposition for the estimation and spatialization of the radiation fluxes in the Amazon region, which may contribute to a better understanding of the interaction between Amazon rainforest and atmosphere, and generate input information needed to the surface models coupled to atmospheric general circulation models.

KEYWORDS: surface net radiation, albedo, remote sensing, SEBAL model, radiometers. 


\section{INTRODUÇÃO}

A Amazônia brasileira passa por uma exploração em grande escala de seus recursos naturais e as diferentes transformaçóes ocorridas na cobertura da superfície, principalmente pela substituição de áreas de floresta por pastagem, exercem uma grande influência na hidrologia e clima (Nobre et al. 2007; Oliveira et al. 2007; Cardoso et al. 2009).

Desde a década de 80 vêm sendo realizados experimentos meteorológicos na região amazônica (e.g. Amazonian Research Micrometeorological Experiment-ARME; Amazonian Boundary Layer Experiment - ABLE; Anglo Brazilian Amazonian Climate Observational Studs - ABRACOS; Large-Scale Biosphere Atmosphere Experiment in Amazonia - LBA), o que têm permitido determinar a variabilidade temporal, em especial ao longo das estaçôes, da energia disponível para os processos de aquecimento direto da atmosfera pela superfície e da evapotranspiração (Gash et al. 2004; Artaxo 2012). No entanto, mediçôes diretas realizadas em campo geralmente são pontuais e dada a extensão e diversidade da região amazônica, faz-se necessária a estimativa e representação espacial dos fluxos de radiaçáo sob uma escala mais ampla, de modo a melhor detalhar as características da camada limite utilizadas em modelos climáticos e de previsão do tempo (Moraes et al. 2003).

Nesse contexto, a utilização de dados de sensoriamento remoto surge como alternativa viável, pois possibilita a estimativa dos componentes do balanço radiativo com vasta cobertura espaço-temporal. O sensor Moderate Resolution Imaging Spectroradiometer (MODIS), a bordo das plataformas TERRA e AQUA, tem como características: cobertura global, alta resolução radiométrica, intervalos dinâmicos nas bandas do visível, infravermelho próximo, infravermelho médio e infravermelho termal (Rojas et al. 2002), que o tornam um instrumento com grande potencial para este tipo de aplicação.

A aplicaçáo de dados do sensor MODIS para estimativa e avaliaçáo dos fluxos energéticos pode ser realizada por intermédio de modelagem, com entrada de dados extraídos de imagens, como reflectância da superfície, temperatura, emissividade e índices de vegetação. O modelo Surface Energy Balance Algorithms for Land (SEBAL) (Bastiaanssen 1995) se destaca pelo fato de utilizar suposiçóes empíricas básicas e por necessitar de dados de sensores orbitais complementados por reduzidas informaçóes de superfície; ademais, tem sido amplamente utilizado e aceito na comunidade científica nacional e internacional (Paiva et al. 2011; Bhattarai et al. 2012).

Diante do exposto, esse estudo tem como objetivo estimar os componentes do balanço de radiação em duas regióes no sudoeste da Amazônia brasileira, a partir de dados MODIS/ TERRA inseridos no modelo SEBAL, e validar os resultados obtidos com informaçóes micrometeorológicas adquiridas pelas torres do projeto LBA sob as condiçôes de pastagem e floresta.

\section{MATERIAL E MÉTODOS}

\section{Área de estudo}

A área de estudo compreende duas regiōes específicas situadas na porçáo centro-leste do estado de Rondônia (RO) (Figura 1). Cada regiáo engloba um sítio experimental

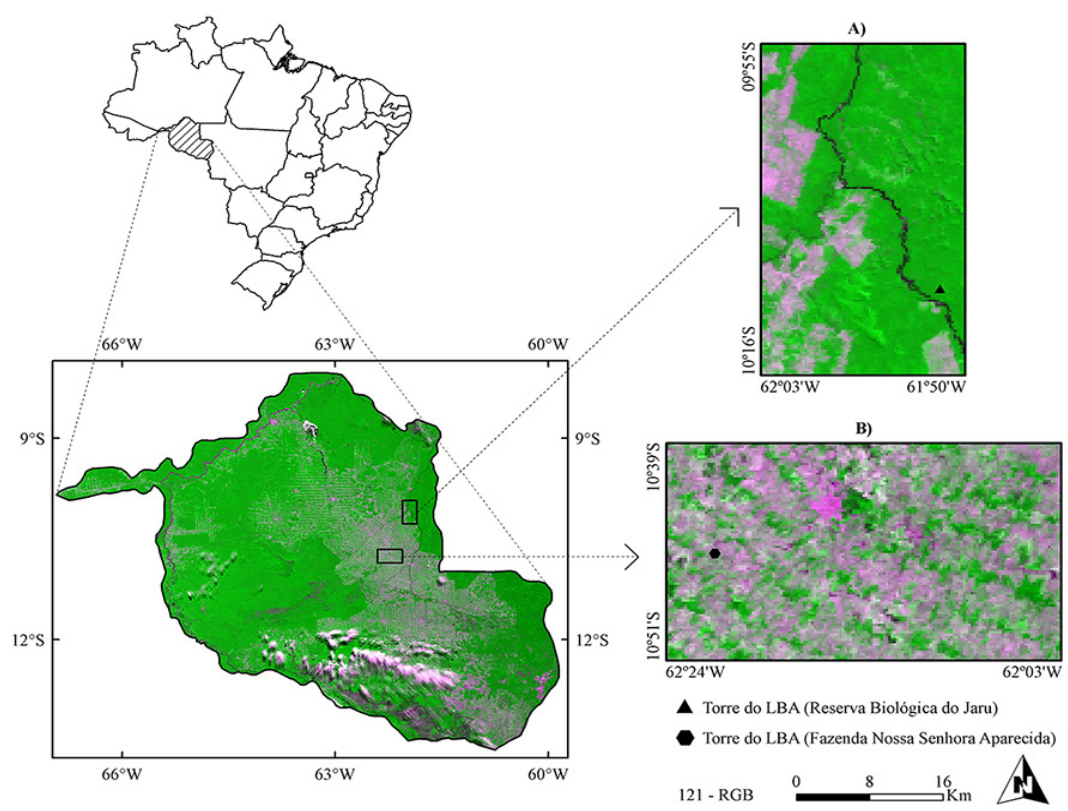

Figura 1 - Localização das regiões de estudo no estado do Rondônia (RO). A) Região da RBJ e B) Região da FNSA. A composição colorida foi obtida com dados adquiridos pelo sensor MODIS/TERRA (MOD09GQ) de 25/07/2003. 
de coleta de dados de superfície (por meio de torres micrometeorológicas) do projeto LBA. Os sítios experimentais são representativos das condiçôes de pastagem e floresta, e estão situados na Fazenda Nossa Senhora Aparecida (FNSA) e na Reserva Biológica do Jaru (RBJ), localizadas nos municípios de Ouro Preto do Oeste e Ji-Paraná, respectivamente. Cada regiāo de estudo (FNSA e RBJ) compreende aproximadamente 85.087 ha, e além das características intrínsecas aos sítios experimentais nelas contidos (pastagem e floresta) são verificados outros tipos de uso e cobertura da terra, como corpos de água e áreas de sucessão secundária.

A regiáo da FNSA engloba parte dos municípios de JiParaná, Nova União, Ouro Preto do Oeste e Teixeirópolis. A maior parte da regiáo é composta por pastagem (74\%), enquanto o restante se refere a áreas de sucessão secundária (16\%) e floresta ombrófila aberta (10\%) (Oliveira 2012). Especificamente em relação ao sítio experimental da FNSA a vegetação predominante é do tipo gramínea (Urochloa brizantha), cuja altura varia de acordo com a estação do ano (Zanchi et al. 2009). A torre micrometeorológica possui $10 \mathrm{~m}$ de altura e situa-se nas coordenadas $10^{\circ} 45^{\prime} 45^{\prime \prime} \mathrm{S}$ e $62^{\circ} 21^{\prime}$ 29" W, a aproximadamente $293 \mathrm{~m}$ acima do nível do mar. Maiores detalhes sobre o sítio da FNSA podem ser encontrados no estudo de Culf et al. (1995).

A região da RBJ compreende parte dos municípios de Ariquemes, Ji-Paraná, Ouro Preto do Oeste, Theobroma e Vale do Paraíso, e é composta principalmente por floresta ombrófila aberta, que ocupa $66 \%$ da região. Os demais tipos de uso e cobertura da terra dizem respeito a áreas de pastagem (22\%), sucessão secundária (10\%) e corpos de água (2\%) (Oliveira 2012). O sítio da RBJ é caracterizado pela presença de floresta tropical úmida, cuja altura média do dossel é de $30 \mathrm{~m}$ (Wright et al. 1996). A torre micrometeorológica possui $64 \mathrm{~m}$ de altura e está situada nas coordenadas $10^{\circ} 11^{\prime} 11^{\prime \prime} \mathrm{S}$ e $61^{\circ} 52^{\prime} 20^{\prime \prime} \mathrm{W}$, a $120 \mathrm{~m}$ de altitude. Informaçôes adicionais com relação a este sítio podem ser encontradas em Von Randow et al. (2004).

\section{Dados micrometeorológicos}

Os dados meteorológicos de superfície foram obtidos das torres micrometeorológicas localizadas nos sítios experimentais de pastagem (FNSA) e floresta (RBJ) sob o âmbito do projeto LBA. As medidas in situ foram utilizadas tanto para inserção direta no modelo SEBAL, como para análise e validação das estimativas para as regiôes da FNSA e RBJ nos dias 01/08/2003 e 05/07/2005 (ambos referentes ao período seco), respectivamente. Na Tabela 1 estáo relacionadas as variáveis meteorológicas usadas com os respectivos instrumentos utilizados em cada torre.

Os arquivos contendo as diversas variáveis meteorológicas possuíam dados correspondentes às médias de 30 minutos, totalizando 48 períodos de mediçóes por dia. Primeiramente
Tabela 1 - Variáveis medidas, instrumentação e altura de instalação dos sensores nas torres dos sítios da FNSA e RBJ.

\begin{tabular}{lccc}
\hline Variável meteorológica & Instrumento & $\begin{array}{c}\text { Altura (m) } \\
\text { FNSA }\end{array}$ & $\begin{array}{c}\text { Altura (m) } \\
\text { RBJ }\end{array}$ \\
\hline $\begin{array}{l}\text { Radiação solar } \\
\text { incidente e refletida }\end{array}$ & $\begin{array}{c}\text { Piranômetro Kipp \& } \\
\text { Zonen (CM21) }\end{array}$ & 6,4 & 57,5 \\
$\begin{array}{l}\text { Radiação infravermelha } \\
\text { termal incidente e } \\
\text { emitida }\end{array}$ & $\begin{array}{c}\text { Pirgeômetro Kipp \& } \\
\text { Zonen (CG1) }\end{array}$ & 6,4 & 57,5 \\
$\begin{array}{l}\text { Temperatura do ar } \\
\text { Termohigrômetro }\end{array}$ & 8,0 & 61,5 \\
Vaisala (HMP35A) & 8,0 & \\
\hline & $\begin{array}{c}\text { Pluviômetro EM } \\
\text { (ARG-100) }\end{array}$ & 0,9 & 61,5 \\
\hline
\end{tabular}

esses dados foram analisados, com o objetivo eliminar dados espúrios, e, posteriormente, tratados estatisticamente. Isto foi necessário devido à existência de problemas nos sensores dos instrumentos de medida nas torres ou erros humanos, visto que se verificaram em alguns horários específicos a ausência de dados/mediçôes. O tratamento estatístico para o preenchimento dessas falhas consistiu na aplicação da metodologia descrita em Lasslop et al. (2010). Após o tratamento dos dados, foram calculados os valores semihorários de albedo (entre as $7 \mathrm{~h} \mathrm{e} 17 \mathrm{~h}$ ) para as duas datas em ambos os sítios.

\section{Dados orbitais}

Foram utilizados dados orbitais MODIS/TERRA referentes aos dias 01/08/2003 (instante de passagem: $10 \mathrm{~h} 31 \mathrm{~min} 38 \mathrm{~s}$ ) e 05/07/2005 (instante de passagem: $10 \mathrm{~h} 31 \mathrm{~min} 44 \mathrm{~s})$. Cabe salientar que para a escolha das datas levou-se em consideração os seguintes fatores: disponibilidade de dados de superfície do LBA, ausência (ou mínima presença) de nebulosidade nas imagens MODIS/TERRA, ausência de precipitação em no mínimo quinze dias antes da coleta dos dados orbitais. Os dados/produtos MODIS/TERRA utilizados neste trabalho corresponderam aos Tiles H11V09 e H11V10 e foram os seguintes: MOD09GQ - reflectância da superfície nas bandas 1 e 2 (vermelho e infravermelho próximo) com resolução espacial de $250 \mathrm{~m}$; MOD09GA - reflectância da superfície nas bandas 3 a 7 (azul, verde e infravermelho médio) com resolução espacial de $500 \mathrm{~m}$; e MOD11A1 - temperatura da superfície (K) com resolução espacial de $1 \mathrm{~km}$.

Os dados MODIS/TERRA foram inicialmente tratados no aplicativo MODIS Reprojection Tool (https://lpdaac.usgs. gov/tools/modis_reprojection_tool), onde foram efetuadas operaçóes de reprojeçáo e mosaicagem. Os dados foram reprojetados para coordenadas geográficas, datum World Geodetic System 84 (WGS84), e então convertidos para o 
formato GeoTiff. Através do programa ENVI 4.5 foi realizada a reamostragem dos pixels dos produtos MOD09GA e MOD11A1 para $250 \mathrm{~m}$, de modo a padronizar a resoluçáo espacial das estimativas. Após o processamento das imagens MODIS/TERRA definiu-se os retângulos envolventes de cada regiáo (FNSA e RBJ) e, posteriormente, efetuou-se as operaçôes de mascaramento de nuvens, que consistiram na identificação visual dessas áreas e delimitação por digitalização ponto a ponto.

\section{Implementação do modelo SEBAL}

O balanço de radiação para o momento de passagem do sensor MODIS/TERRA $(\approx 10 \mathrm{~h} 30 \mathrm{~min})$ foi estimado partindose dos valores de reflectância in-band da superfície contidos nos dados/produtos MOD09 conforme descrito a seguir (Bastiaanssen 1995):

$$
\left(1-\alpha_{\mathrm{s}}\right) \mathrm{K} \downarrow+\mathrm{L} \downarrow-\mathrm{L} \uparrow-\left(1-\varepsilon_{\mathrm{s}}\right) \mathrm{L} \downarrow
$$

em que Rn é o balanço de radiação na superfície ( $\left.\mathrm{W} \mathrm{m}^{-2}\right)$, $\alpha_{\mathrm{s}}$ é o albedo da superfície, $K \downarrow$ representa a radiação solar incidente na superfície ( $\left.\mathrm{W} \mathrm{m}^{-2}\right), \mathrm{L} \downarrow$ é a radiação infravermelha termal (IVT) incidente na superfície ( $\left.\mathrm{W} \mathrm{m}^{-2}\right), \mathrm{L} \uparrow$ é a radiação IVT emitida pela superfície ( $\mathrm{W} \mathrm{m}^{-2}$ ) e $\varepsilon_{\mathrm{s}}$ é a emissividade da superfície.

As estimativas de albedo foram realizadas de acordo com Liang (2001):

$\alpha=0,160 \alpha_{1}+0,291 \alpha-0,243 \alpha_{3}-0,116 \alpha_{4}-0,112 \alpha_{5}+0,081 \alpha_{7}-0,0015$

em que $\alpha_{\mathrm{s}}$ é o albedo da superfície e $\alpha_{\mathrm{i}}(\mathrm{i}=1$ a 7 ) é a reflectância in-band da superfície correspondente as bandas 'i' do sensor MODIS.

Para o cálculo da emissividade da superfície, primeiramente foi necessário calcular três índices de vegetação: o índice de vegetação por diferença normalizada (NDVI), o índice de vegetação ajustado ao solo (SAVI) e o índice de área foliar (LAI).

O NDVI foi obtido através da razão entre a diferença das reflectâncias nas faixas do infravermelho próximo e do vermelho pela soma das mesmas (Equação 3):

$$
\text { NDVI }=\frac{\rho_{2}-\rho_{1}}{\rho_{2}+\rho_{1}}
$$

em que $\rho_{1}$ e $\rho_{2}$ são as reflectâncias nas bandas 1 e 2 do sensor MODIS.

Para o cálculo do SAVI (Huete 1988), que é um índice que busca amenizar os efeitos do solo no NDVI, foi utilizada a Equação 4:

$$
(1+\delta)\left(\rho_{2}-\rho_{1}\right) /\left(\delta+\rho_{2}+\rho_{1}\right)
$$

em que $\delta$ é uma constante de ajuste, cujo valor mais comumente referenciado é 0,5 (Huete 1988;
Boegh et al. 2002). Portanto, também foi adotado nesta pesquisa.

Após o cálculo do NDVI e do SAVI, calculou-se o LAI através da Equação 5 (Allen et al. 2002).

$$
\mathrm{LAI}=\frac{\ln \left(\frac{0,69-S A V I}{0,59}\right)}{0,91}
$$

Assim sendo, para o cômputo da emissividade da superfície a partir dos índices de vegetação, Allen et al. (2002) propuseram a aplicação da equação:

$0,95+0,01 \Lambda \mathrm{AI}$

A Equação 6, porém, só é válida para os pixels que apresentarem NDVI maior que zero. Para os pixels com NDVI negativo, adotou-se $\varepsilon_{\mathrm{s}}=0,985$ (Allen et al. 2002).

A radiação solar incidente foi calculada através da expressão:

$$
\mathrm{K} \downarrow=\tau \mathrm{S}_{0}\left(\frac{\mathrm{d}_{\mathrm{m}}}{\mathrm{d}}\right)^{2} \cos \theta_{\mathrm{z}}
$$

em que $S_{0}$ corresponde a constante solar $\left(1367 \mathrm{~W} \mathrm{~m}^{-2}\right), \theta_{\mathrm{z}}$ ao ângulo zenital solar (radianos) ( 0,673 e 0,717 radianos para os pixels centrais dos recortes em 01/08/2003 e 05/07/2005, respectivamente), $\mathrm{d}_{\mathrm{m}}$ e d são, respectivamente, as distâncias média e instantânea entre a Terra e o Sol e $\tau$ corresponde a transmitância da atmosfera no instante da passagem do satélite.

O cálculo da transmitância atmosférica se deu de acordo com Allen et al. (2007):

$$
\tau=0,75+2 \times 10^{-5} \mathrm{z}
$$

em quezéa altitude (m) no pixelda torre micrometeorológica utilizada para entrada de dados do SEBAL.

A radiação IVT incidente na superfície foi computada a partir da equação de Stefan-Boltzmann (Santos et al. 2011b):

$$
\mathrm{L} \downarrow=\varepsilon_{\mathrm{a}} \sigma \mathrm{T}_{\mathrm{a}}^{4}
$$

em que $\varepsilon_{a}$ é a emissividade da atmosfera, $\sigma$ é a constante de Stefan-Boltzmann $\left(5,67 \times 10^{-8} \mathrm{~W} \mathrm{~m}^{-2} \cdot \mathrm{K}^{-4}\right)$ e $\mathrm{T}_{\mathrm{a}}$ é a temperatura do ar próxima à superfície $(\mathrm{K})$.

Como visto na Equação 9, um dos termos que se insere na equação de Stefan-Boltzmann é a emissividade da atmosfera, que neste estudo foi assim calculada:

$$
\varepsilon_{\mathrm{a}}=0,85(-\ln \tau)^{0,09}
$$

$\mathrm{O}$ cômputo da radiaçăo IVT emitida pela superfície foi obtido utilizando-se a equação de Stefan-Boltzmann:

$\mathrm{L} \uparrow=\varepsilon_{s} \sigma \mathrm{T}_{s}^{4}$

em que $\mathrm{T}_{\mathrm{s}}$ é a temperatura da superfície (K).

Após os procedimentos para a obtenção do balanço de radiação para o instante da passagem do satélite, calculou- 
se o balanço de radiação médio diário a partir da expressão proposta por Bastiaanssen et al. (2005):

$$
R n_{24 h}=\left(1-\alpha_{s}\right) K \downarrow_{24 h}-110 \tau
$$

em que $\mathrm{K} \downarrow_{24 \mathrm{~h}}$ é a média diária de radiação solar incidente na superfície $\left(\mathrm{W} \mathrm{m}^{-2}\right)$ e 110 é um valor constante, expresso em W m ${ }^{-2}$.

\section{Validação das estimativas}

De forma a avaliar a concordância entre os valores observados e modelados foram calculados o viés (VS) e o erro relativo (ER), descritos de acordo com as Equaçôes 13 e 14.

$$
\begin{aligned}
& \mathrm{VS}=\left(\mathrm{F}_{\text {mod }}-\mathrm{F}_{\text {obs }}\right) \\
& \mathrm{ER}=100\left(\frac{\left|\mathrm{F}_{\text {mod }}-\mathrm{F}_{\text {obs }}\right|}{\mathrm{F}_{\text {obs }}}\right)
\end{aligned}
$$

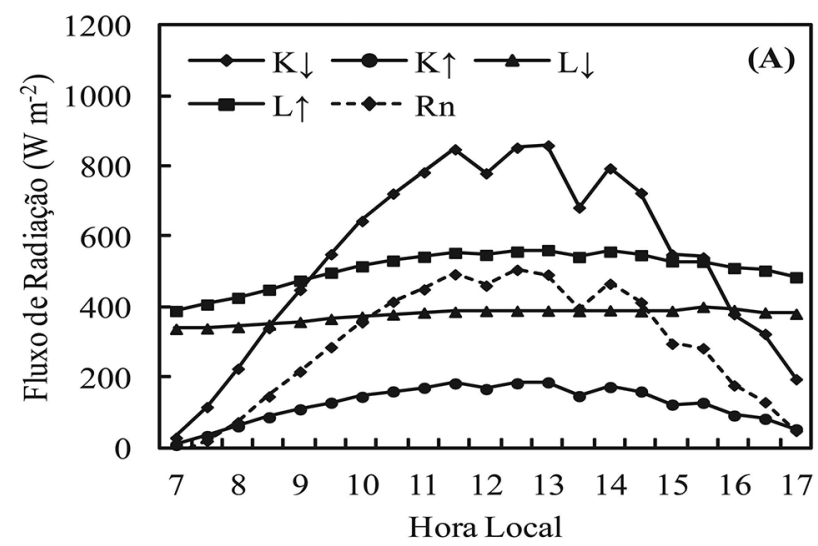

em que $\mathrm{F}_{\bmod }$ representa a medida do fluxo estimada pelo modelo SEBAL e $\mathrm{F}_{\text {obs }}$ representa a medida observada no pixel da torre micrometeorológica.

\section{RESULTADOS}

\section{Comportamento dos componentes do balanço de radiação nos sítios de pastagem (FNSA) e floresta (RBJ)}

As Figuras 2 e 3 mostram os fluxos de radiaçáo e o albedo medidos no período diurno ( $7 \mathrm{~h}$ às $17 \mathrm{~h}$ ) pelas torres micrometeorológicas nos sítios da FNSA (01/08/2003) e RBJ (05/07/2005), respectivamente.

Ao analisar a variação de $\mathrm{K} \downarrow$ nos sítios da FNSA e RBJ, verifica-se que praticamente não houve interferência de nuvens ao longo dos dias avaliados. Especificamente, observou-se a presença de nebulosidade no sítio da FNSA

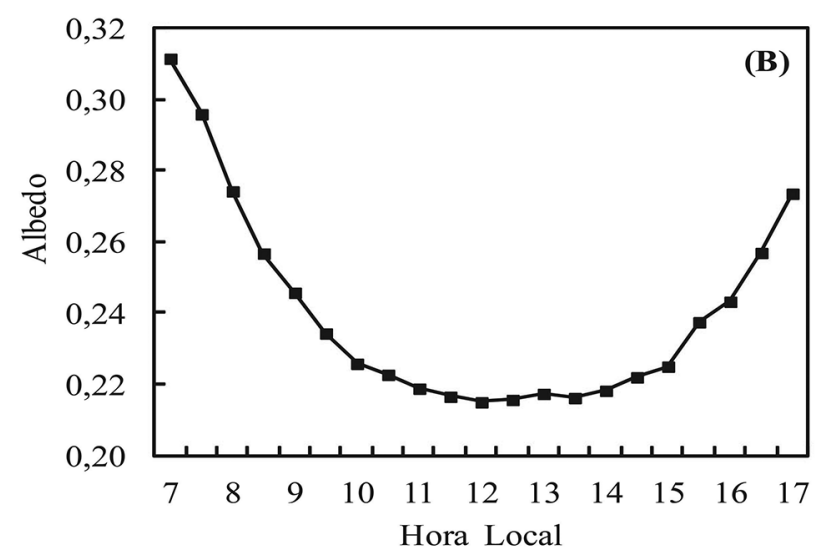

Figura 2 - Medidas dos componentes do balanço de radiação durante o período diurno na torre micrometeorológica da FNSA em 01/08/2003. (A) Fluxos de radiação (radiação solar incidente $(\mathrm{K} \downarrow)$, radiação solar refletida $(\mathrm{K} \uparrow)$, radiação IVT incidente $(\mathrm{\downarrow} \downarrow)$, radiação IVT emitida $(\mathrm{L} \uparrow)$ e balanço de radiação $(\mathrm{Rn})$ ) e $(\mathrm{B})$ albedo.
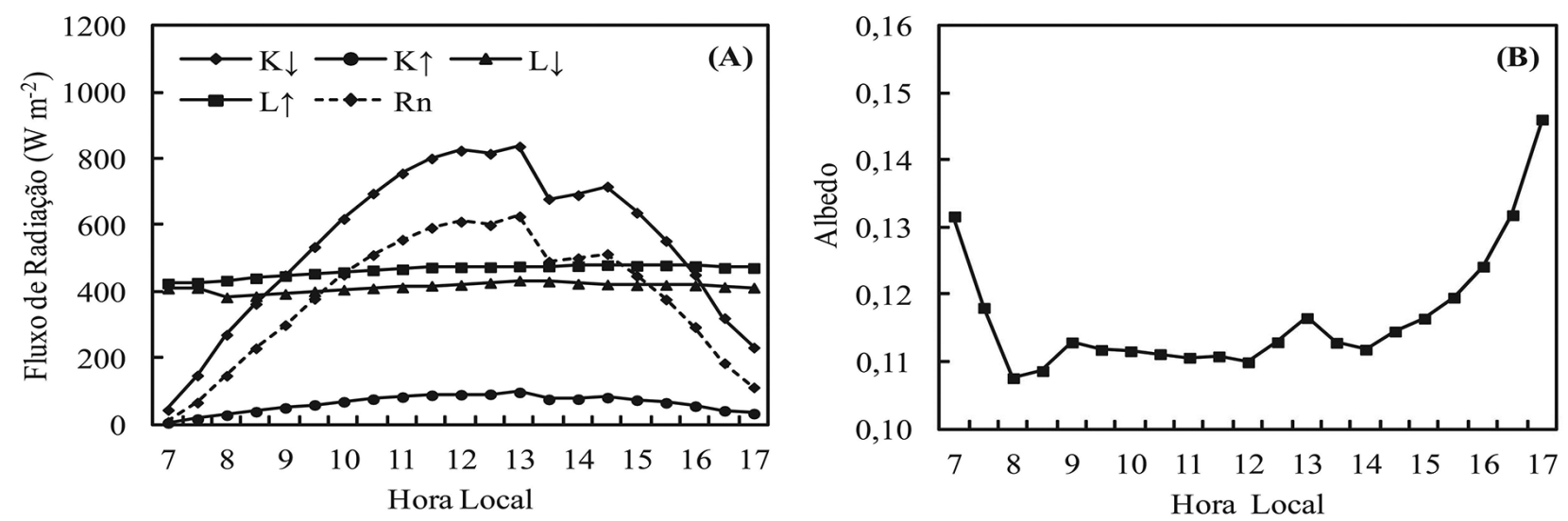

Figura 3 - Medidas dos componentes do balanço de radiação durante o período diurno na torre micrometeorológica da RBJ em 05/07/2005. (A) Fluxos de radiação (radiação solar incidente $(\mathrm{K} \downarrow)$, radiação solar refletida $(\mathrm{K} \uparrow)$, radiação IVT incidente $(\mathrm{L} \downarrow)$, radiação IVT emitida $(\mathrm{L} \uparrow)$ e balanço de radiação $(\mathrm{Rn})$ ) e $(\mathrm{B})$ albedo. 
às $12 \mathrm{~h}$ e $13 \mathrm{~h} 30 \mathrm{~min}$, e no sítio da RBJ às $13 \mathrm{~h} 30 \mathrm{~min}$. Em ambos os sítios os maiores registros de $\mathrm{K} \downarrow$ ocorreram às $13 \mathrm{~h}$, apresentando valores máximos de $859,0 \mathrm{~W} \mathrm{~m}^{-2}$ (FNSA) e $837,0 \mathrm{~W} \mathrm{~m}^{-2}$ (RBJ). A tendência da variação de $\mathrm{K} \uparrow$ foi semelhante na pastagem e na floresta, com valores máximos ocorrendo próximos às $13 \mathrm{~h}$, acompanhando a variação de $\mathrm{K} \downarrow$. Os resultados relacionados à $\mathrm{K} \downarrow$ e $\mathrm{K} \uparrow$ estáo de acordo com o estudo de Galvão e Fisch (2000), que analisaram essas variáveis para dias relativos à estaçáo seca do ano de 1993 nos sítios da FNSA e RBJ. A média de K $\uparrow$ para o período diurno foi de $123,5 \mathrm{~W} \mathrm{~m}^{-2}$ em 01/08/2003 na FNSA e de $62,4 \mathrm{~W} \mathrm{~m}^{-2} \mathrm{em}$ 05/07/2005 na RBJ, evidenciando o fato de que a floresta absorve mais a radiação solar que a pastagem.

Nos dois sítios, durante o período diurno, a $\mathrm{L} \downarrow$ foi inferior a $L \uparrow$, no entanto, verificou-se que ao longo do período diurno há uma maior amplitude entre os valores de $\mathrm{L} \downarrow$ e $\mathrm{L} \uparrow$ no sítio da FNSA. A média de L $\downarrow$ na FNSA foi de $373,4 \mathrm{~W} \mathrm{~m}^{-2} \mathrm{e}$ na RBJ de 415,0 $\mathrm{W} \mathrm{m}^{-2}$, enquanto a média de $\mathrm{L} \uparrow$ na FNSA correspondeu a 507,9 W m $\mathrm{W}^{-2}$ e na RBJ a 463,2 $\mathrm{W} \mathrm{m}^{-2}$. Assim, a $\mathrm{L} \uparrow$ foi em média $9,7 \%$ maior na pastagem do que na floresta, enquanto a $\mathrm{L} \downarrow$ foi em média $11,1 \%$ superior na floresta. $\mathrm{O} \mathrm{Rn}$ (tanto na floresta como na pastagem) acompanhou as variaçóes de $\mathrm{K} \downarrow$, que representa o componente de maior influência no balanço de radiaçáo. Os máximos valores de Rn encontrados na FNSA e na RBJ foram de 493,2 W m ${ }^{-2}$ (11h30min) e 626,9 W m ${ }^{-2}$ (13h), respectivamente. Na média o Rn na pastagem foi $23,6 \%$ inferior ao da floresta, resultado que concorda com os estudos de Culf et al. (1996) e Aguiar (2007).

Percebe-se que em ambos os sítios os maiores valores de albedo ocorreram nas primeiras horas da manhã e no final da tarde, devido à dependência desta variável com o ângulo zenital solar (Souza et al. 2010). O albedo médio diário do período foi 0,24 na pastagem e 0,12 na floresta e está de acordo com os valores encontrados por Von Randow et al. (2004).

\section{Estimativas para a região da FNSA}

As Figuras 4 e 5 ilustram, respectivamente, a distribuição espacial do albedo e do balanço de radiaçáo $(\mathrm{Rn})$ na regiáo da FNSA no dia 01/08/2003.

Os valores mínimos e máximos de albedo obtidos a partir de dados MODIS/TERRA foram 0,088 e 0,234, respectivamente, e o valor médio da imagem gerada correspondeu a 0,173. Verifica-se na Figura 4 que os menores valores de albedo se referiram às áreas de floresta ombrófila aberta, cujos valores variaram entre $0,088 \mathrm{e} \approx 0,15$. A título de comparação, Liberato (2011), em um estudo no estado de RO, utilizando dados TM/Landsat 5, encontrou valores de albedo em áreas de floresta nativa variando entre 0,12 e 0,13. As áreas de sucessão secundária apresentaram valores entre $\approx 0,15$ e $\approx 0,17$, e as áreas de pastagem apresentaram valores geralmente superiores a 0,17 . Os valores obtidos concordam com o estudo de Santos et al. (2011a), que encontraram para áreas de pastagem em RO, valores médios de albedo de 0,19 e 0,22 para dias referentes ao período seco de 2008 .

$\mathrm{Na}$ imagem de $\mathrm{Rn}$ os valores variaram entre 478,6 e $594,7 \mathrm{~W} \mathrm{~m}^{-2}$, sendo o valor médio igual a $528,2 \mathrm{~W} \mathrm{~m}^{-2}$. Pode se observar na Figura 5 que os maiores valores de $\mathrm{Rn}$ corresponderam as áreas de floresta ombrófila aberta, apresentando valores quase sempre superiores a $539 \mathrm{~W} \mathrm{~m}^{-2}$. As áreas de sucessão secundária apresentaram valores em torno de 528 a $539 \mathrm{~W} \mathrm{~m}^{-2}$, e as áreas de pastagem apresentaram valores $\leq 528 \mathrm{~W} \mathrm{~m}^{-2}$. Estudos utilizando dados TM/Landsat 5 e o modelo SEBAL em RO mostraram valores médios de Rn para áreas de floresta nativa, de 592 e $607 \mathrm{~W} \mathrm{~m}^{-2}$ (Santos

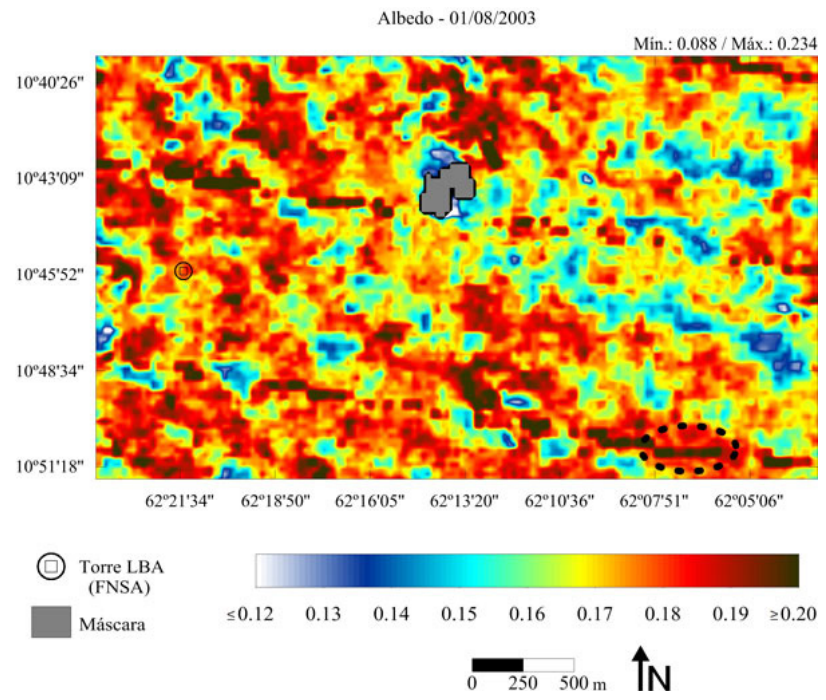

Figura 4 - Distribuição espacial do albedo obtido a partir de dados MODIS/ TERRA na região da FNSA em 01/08/2003.

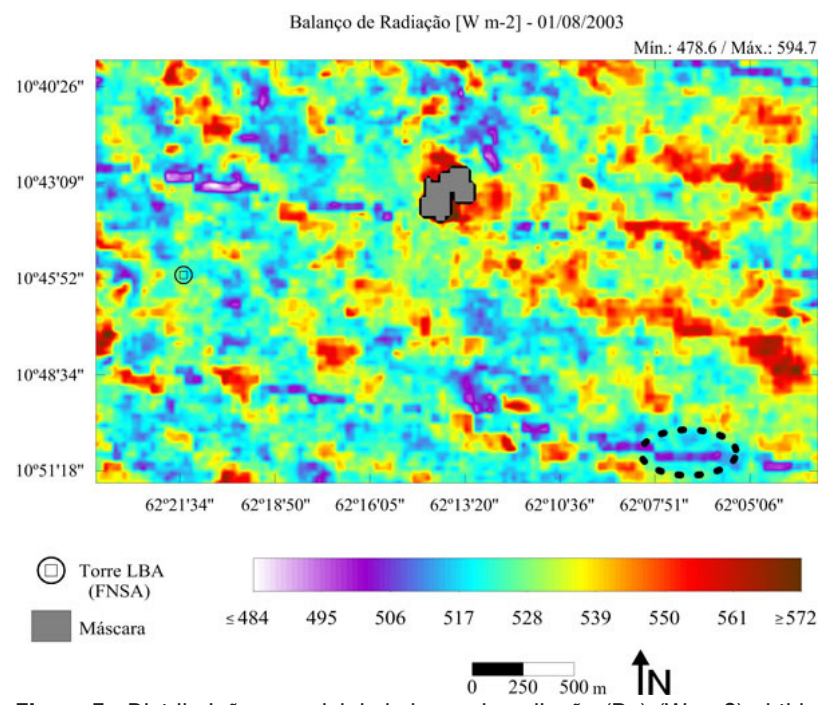

Figura 5 - Distribuição espacial do balanço de radiação (Rn) (W m-2) obtido a partir de dados MODIS/TERRA na regiẫo da FNSA em 01/08/2003. 
Tabela 2 - Medidas de concordância (Viés (VS) (W m²) e Erro Relativo (ER) (\%)) entre os valores modelados (M) pelo SEBAL, a partir de dados MODIS/

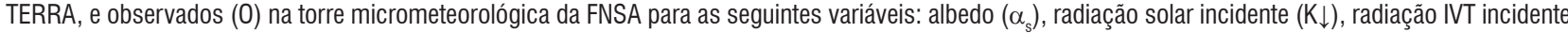
$(L \downarrow)$, radiação IVT emitida $(\mathrm{\uparrow} \uparrow)$, balanço de radiação $(R n)$ e balanço de radiação médio diário $\left(\mathrm{Rn}_{24 h}\right)$.

\begin{tabular}{|c|c|c|c|c|c|c|c|c|c|c|c|c|}
\hline \multicolumn{13}{|c|}{ Região da Fazenda Nossa Senhora Aparecida - 01/08/2003 } \\
\hline & \multicolumn{2}{|c|}{$\alpha_{s}$} & \multicolumn{2}{|c|}{$\mathrm{K} \downarrow\left(\mathrm{W} \mathrm{m}^{-2}\right)$} & \multicolumn{2}{|c|}{$L \downarrow\left(W^{-2}\right)$} & \multicolumn{2}{|c|}{$\mathrm{L} \uparrow\left(\mathrm{W} \mathrm{m}^{-2}\right)$} & \multicolumn{2}{|c|}{$\operatorname{Rn}\left(\mathrm{W} \mathrm{m}^{-2}\right)$} & \multicolumn{2}{|c|}{$\mathrm{Rn}_{24 \mathrm{~h}}\left(\mathrm{~W} \mathrm{~m}^{-2}\right)$} \\
\hline & M & 0 & M & 0 & M & 0 & M & 0 & M & 0 & M & 0 \\
\hline & 0,177 & 0,219 & 784,6 & 782,7 & 398,0 & 384,6 & 501,2 & 543,0 & 524,1 & 450,5 & 114,6 & 111,8 \\
\hline VS & \multicolumn{2}{|c|}{$-0,042$} & \multicolumn{2}{|c|}{1,9} & \multicolumn{2}{|c|}{13,4} & \multicolumn{2}{|c|}{$-41,8$} & \multicolumn{2}{|c|}{73,6} & \multicolumn{2}{|c|}{2,8} \\
\hline ER & \multicolumn{2}{|c|}{19,2} & \multicolumn{2}{|c|}{0,2} & \multicolumn{2}{|c|}{3,5} & \multicolumn{2}{|c|}{7,7} & \multicolumn{2}{|c|}{16,3} & \multicolumn{2}{|c|}{2,5} \\
\hline
\end{tabular}

et al. 2011a), e valores variando entre 386 e $507 \mathrm{~W} \mathrm{~m}^{-2}$ em áreas de pastagem (Liberato 2010).

A Tabela 2 mostra os valores dos componentes de Rn obtidos a partir de dados MODIS/TERRA com base no modelo SEBAL, e os observados na torre micrometeorológica em área de pastagem (sítio da FNSA).

Ao analisar a Tabela 2 denota-se uma excelente à razoável concordância entre os valores modelados e os observados na torre micrometeorológica situada em área de pastagem. Os maiores erros relativos (ERs) foram verificados nas estimativas de albedo e $\mathrm{Rn}$, e os menores nas estimativas de $\mathrm{K} \downarrow$ e $\mathrm{Rn}_{24 \mathrm{~h}}$. O albedo modelado $(0,177)$ apresentou uma razoável concordância com o albedo observado $(0,219)$, representando viés de -0,042 e ER de 19,2\%. A título de comparação, Gomes (2009) encontrou para estimativas de albedo em áreas de cerrado, utilizando imagens TM/Landsat 5, ERs variando entre $0,8 \%$ e $19,6 \%$. Para o Rn verificou-se ER de $16,3 \%$, que se deveu ao fato da estimativa pelo modelo SEBAL $(524,1$ $\mathrm{W} \mathrm{m} \mathrm{m}^{-2}$ ) ter sido $73,6 \mathrm{~W} \mathrm{~m}^{-2}$ superior à medida pela torre micrometeorológica $\left(450,5 \mathrm{~W} \mathrm{~m}^{-2}\right)$. Lira (2008) obteve ERs de $9,2 \%$ e $18,4 \%$ em estimativas de Rn pelo modelo SEBAL em áreas de cana-de-açúcar, e Giongo (2011) obteve ERs de até $31,8 \%$ nas estimativas de Rn para áreas de cerrado.

$\mathrm{O}$ valor de $\mathrm{K} \downarrow$ obtido a partir da imagem MODIS/ TERRA apresentou uma excelente concordância (ER=0,2\%) com o valor de $\mathrm{K} \downarrow$ observado na torre micrometeorológica da FNSA. Silva et al. (2005) obtiveram ER de 3,2\% na estimativa de $\mathrm{K} \downarrow$, a partir de dados TM/Landsat 5, com o dado medido na estação micrometeorológica de Bebedouro (PE). Esses resultados evidenciam que a equação de estimativa de $\mathrm{K} \downarrow$ utilizada no modelo SEBAL apresenta boa acurácia. A estimativa de $\mathrm{Rn}_{24 \mathrm{~h}}$ para a área de pastagem apresentou ER de apenas 2,5\%, superestimando o valor medido pela torre em 2,8 W m ${ }^{-2}$. Essa medida de erro é melhor que as obtidas por Ruhoff et al. (2012), que encontraram para estimativas de $\mathrm{Rn}_{24 \mathrm{~h}}$ utilizando dados MODIS (TERRA e AQUA) ERs de $15 \%$ e $23 \%$.
As estimativas de $\mathrm{L} \downarrow$ e $\mathrm{L} \uparrow$ apresentaram valores bastante próximos às mediçôes in situ, representando, respectivamente, ERs de 3,5\% e 7,7\%, resultados semelhantes aos obtidos por Silva et al. (2005). Salienta-se que a boa precisão da estimativa de L $\uparrow$ evidencia o potencial da utilização de dados MODIS/ TERRA e o modelo SEBAL em estudos envolvendo, por exemplo, a identificação e o mapeamento de áreas queimadas, um dos principais agentes de mudanças de uso e cobertura da terra na Amazônia.

As estimativas de radiação IVT emitida pela superfície e balanço de radiação relacionam-se à emissividade da superfície, que está ligada ao cômputo do SAVI e do LAI (Marx et al. 2008). Este último é, ainda, um parâmetro chave no que diz respeito as trocas de energia e massa no sistema solovegetação-atmosfera, sendo utilizado em diversos modelos globais climáticos e hidrológicos (Wang et al. 2005). Dada a importância desse índice, o ideal seria o ajuste das constantes empíricas do LAI para as condiçóes específicas de estudo, no entanto, não foi possível obter medidas in situ para os dias trabalhados, o que impossibilitou a calibração dessa equação e a consequente validação. Assim, optou-se por utilizar a Equação 5 e, então, comparar as estimativas de LAI com estudos micrometeorológicos já realizados nos sítios do LBA. Para o pixel referente a torre da FNSA no dia 01/08/2003 foi estimado o valor de $0,44 \mathrm{~m}^{2} \mathrm{~m}^{-2}$. Roberts et al. (1996) encontraram para o sítio da FNSA valores para o período seco variando entre 1,55 e 1,66 $\mathrm{m}^{2} \mathrm{~m}^{-2}$, e Zanchi et al. (2009) obtiveram valores para os períodos secos de 2000 e 2001 entre 1,1 e $1,3 \mathrm{~m}^{2} \mathrm{~m}^{-2}$. Como se verifica, os valores modelados foram subestimados, em média, em $0,96 \mathrm{~m}^{2} \mathrm{~m}^{-2}$.

\section{Estimativas para a região da $\mathrm{RBJ}$}

As Figuras 6 e 7 apresentam, respectivamente, a distribuição espacial do albedo e do balanço de radiação $(R n)$ na regiấo da RBJ no dia 05/07/2005.

Os valores mínimos e máximos da imagem de albedo estimada por dados MODIS/TERRA na região da RBJ foram de 0,052 e 0,189 , respectivamente. $\mathrm{O}$ valor médio foi 

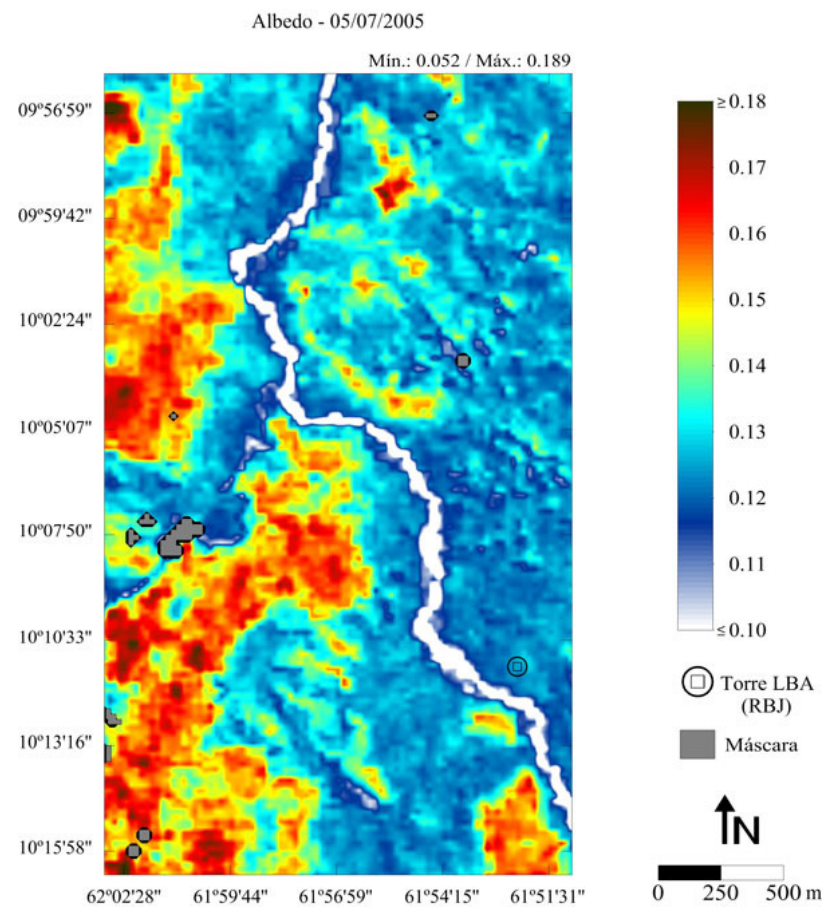

Figura 6 - Distribuição espacial do albedo obtido a partir de dados MODIS/ TERRA na região da RBJ em 05/07/2005.

de 0,133 , e ao comparar com o valor médio da imagem de albedo da regiáo da FNSA $(0,173)$, verifica-se uma diferença de $-23,1 \%$, devida principalmente ao fato da regiáo da RBJ ser composta em sua maior parte por áreas de floresta $(\approx 66 \%)$, ao contrário da regiáo da FNSA, que apresenta prioritariamente áreas de pastagem $(\approx 74 \%)$. Observa-se na imagem de albedo que os menores valores se referiram aos corpos de água $(\leq 0,10)$ e os maiores às áreas de pastagem $(\geq 0,16)$. As áreas de floresta ombrófila aberta compreenderam valores entre $\approx 0,10$ e 0,14 , e as áreas de sucessão secundária valores geralmente entre $\approx 0,14$ e 0,16 .

A imagem de $\mathrm{Rn}$ apresentou valor mínimo de 488,8 $\mathrm{W} \mathrm{m}{ }^{-2}$, médio de $537,8 \mathrm{~W} \mathrm{~m}^{-2}$, e máximo de $601,3 \mathrm{~W} \mathrm{~m}^{-2}$. Percebe-se que o valor médio obtido foi $1,8 \%$ superior ao valor médio da imagem de Rn para a região da FNSA (528,2

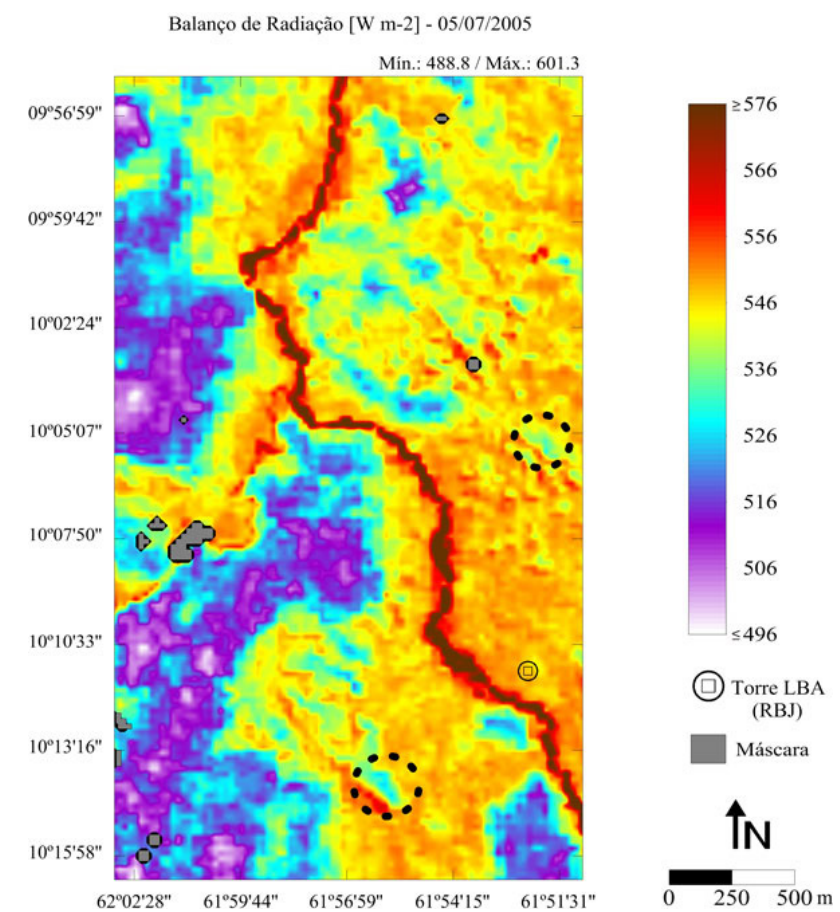

Figura 7 - Distribuição espacial do balanço de radiação (Rn) (W m-2) obtido a partir de dados MODIS/TERRA na região da RBJ em 05/07/2005.

$\mathrm{W} \mathrm{m}^{-2}$ ). Isso se deu pelo fato da regiáo da $\mathrm{RBJ}$ possuir maior quantidade de áreas de floresta, que estáo relacionadas a menores valores de albedo e maiores valores de $\mathrm{Rn}$. Ao visualizar a Figura 7 observa-se que é possível discriminar com facilidade os corpos de água e as áreas de floresta ombrófila aberta das demais classes de uso e cobertura da terra. No entanto, a discriminação entre as áreas de sucessão secundária e pastagem é mais difícil devido à proximidade, por vezes, dos valores estimados para essas áreas. De modo geral, os maiores valores de Rn estiveram associados aos corpos de água, e os menores às áreas de pastagem. Os corpos de água apresentaram valores geralmente superiores a $556 \mathrm{~W} \mathrm{~m}^{-2}$, enquanto as áreas de pastagem mostraram valores quase sempre inferiores a 516 $\mathrm{W} \mathrm{m}{ }^{-2}$. Nas áreas de floresta ombrófila aberta os menores valores foram de $\approx 536 \mathrm{~W} \mathrm{~m}^{-2}$, e os maiores de $\approx 556 \mathrm{~W} \mathrm{~m}^{-2}$.

Tabela 3 - Medidas de concordância (Viés (VS) (W m²-2) e Erro Relativo (ER) (\%)) entre os valores modelados (M) pelo SEBAL, a partir de dados MODIS/

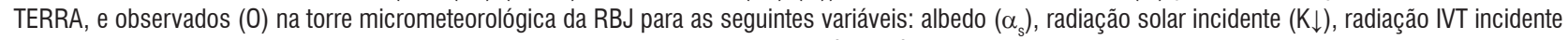
$(\mathrm{L} \downarrow)$, radiação IVT emitida $(\mathrm{L} \uparrow)$, balanço de radiação $(\mathrm{Rn})$ e balanço de radiação médio diário $\left(\mathrm{Rn}_{24 \mathrm{~h}}\right)$.

\begin{tabular}{|c|c|c|c|c|c|c|c|c|c|c|c|c|}
\hline \multicolumn{13}{|c|}{ Região da Reserva Biológica do Jaru - 05/07/2005 } \\
\hline & \multicolumn{2}{|c|}{$\alpha_{s}$} & \multicolumn{2}{|c|}{$\mathrm{K} \downarrow\left(\mathrm{W} \mathrm{m}^{-2}\right)$} & \multicolumn{2}{|c|}{$\mathrm{L} \downarrow\left(\mathrm{W} \mathrm{m}^{-2}\right)$} & \multicolumn{2}{|c|}{$\mathrm{L} \uparrow\left(\mathrm{W} \mathrm{m}^{-2}\right)$} & \multicolumn{2}{|c|}{$\operatorname{Rn}\left(\mathrm{W} \mathrm{m}^{-2}\right)$} & \multicolumn{2}{|c|}{$\mathrm{Rn}_{24 h}\left(\mathrm{~W} \mathrm{~m} \mathrm{~m}^{-2}\right)$} \\
\hline & $M$ & 0 & $M$ & 0 & $M$ & 0 & $M$ & 0 & $M$ & 0 & $M$ & 0 \\
\hline & 0,125 & 0,111 & 749,7 & 756,0 & 357,1 & 412,9 & 452,4 & 467,7 & 547,3 & 556,6 & 128,1 & 151,8 \\
\hline VS & \multicolumn{2}{|c|}{0,014} & \multicolumn{2}{|c|}{$-6,3$} & \multicolumn{2}{|c|}{$-55,8$} & \multicolumn{2}{|c|}{$-15,3$} & \multicolumn{2}{|c|}{$-9,3$} & \multicolumn{2}{|c|}{$-23,7$} \\
\hline ER & \multicolumn{2}{|c|}{12,6} & \multicolumn{2}{|c|}{0,8} & \multicolumn{2}{|c|}{13,5} & \multicolumn{2}{|c|}{3,3} & \multicolumn{2}{|c|}{1,7} & \multicolumn{2}{|c|}{15,6} \\
\hline
\end{tabular}


A Tabela 3 apresenta os valores dos componentes de $\mathrm{Rn}$ obtidos a partir de dados MODIS/TERRA com base no SEBAL, e os observados na torre micrometeorológica em área de floresta (sítio da RBJ).

A Tabela 3 mostra uma concordância aceitável para a generalidade das situaçóes, verificando-se, no entanto, algumas discrepâncias na comparação com as mediçôes realizadas in situ na área de floresta, principalmente com relação à $\mathrm{Rn}_{24 \mathrm{~h}}$ e $\mathrm{L} \downarrow$. Ao considerar o ER, observa-se que as estimativas menos precisas foram de $\mathrm{Rn}_{24 \mathrm{~h}}$ e $\mathrm{L} \downarrow$, enquanto as mais precisas disseram respeito à $\mathrm{K} \downarrow \mathrm{e} \mathrm{Rn}$. $\mathrm{O} \mathrm{Rn}_{24 \mathrm{~h}}$ modelado pelo SEBAL foi inferior ao valor medido em campo em 23,7 W m ${ }^{-2}$, o que acarretou no maior ER $(15,6 \%)$ dentre todas as estimativas para a área de floresta. A $\mathrm{L} \downarrow$ obtida a partir de dados MODIS/TERRA $\left(357,1 \mathrm{~W} \mathrm{~m}^{-2}\right)$ foi inferior à medida pela torre micrometeorológica do sítio da RBJ $(412,9 \mathrm{~W}$ $\mathrm{m}^{-2}$ ), ocasionando um ER de 13,5\%. Esse ER foi 10 pontos percentuais superior ao observado na estimativa dessa variável para o sítio de pastagem na regiáo da FNSA (3,5\%), o que demonstra que a $\mathrm{L} \downarrow$ foi melhor estimada para essa condição.

$A \mathrm{~K} \downarrow$ foi a que apresentou a melhor concordância com o dado da torre (situação análoga à observada para a região da FNSA) com ER $<1 \%$. A estimativa do Rn foi muito próxima à observada em campo, com ER de 1,7\%, denotando que o SEBAL modelou mais satisfatoriamente essa variável na área de floresta nativa do que na área de pastagem (onde se encontrou um ER de 16,3\%). As estimativas de albedo e $\mathrm{L} \uparrow$ para a área de floresta apresentaram melhores concordâncias que as mesmas estimativas para a área de pastagem (sítio da FNSA), apresentando ERs de 12,6\% e 3,3\%.

$\mathrm{O}$ valor modelado de LAI para floresta (pixel da torre da RBJ) foi de $1,16 \mathrm{~m}^{2} \mathrm{~m}^{-2}$, aproximadamente quatro vezes inferior ao observado por Roberts et al. (1996) em um estudo micrometeorológico no sítio da RBJ $\left(4,6 \mathrm{~m}^{2} \mathrm{~m}^{-2}\right)$.

\section{DISCUSSÃO}

As medidas de fluxos de superfície obtidas pelas torres do LBA nos sítios da FNSA e RBJ demonstraram que nos dias analisados (01/08/2003 e 05/07/2005) praticamente não houve interferência de nuvens (sendo estes considerados dias de céu-claro), e que os valores medidos foram concordantes com outros estudos micrometeorológicos realizados nestes sítios, evidenciando a qualidade das observações nas duas datas analisadas.

Foi verificada, tanto nas imagens da regiáo da FNSA quanto da regiáo da RBJ, uma relação de caráter inverso entre $\mathrm{Rn}$ e albedo, onde áreas com maiores valores de Rn estiveram associadas a menores valores de albedo, e vice-versa. Esse aspecto deixa clara a atuação das propriedades da superfície na reflexáo da radiaçấo solar e sua relaçáo com o total de energia disponibilizado aos processos de aquecimento do ar, aquecimento do solo e evaporaçáo. Também se acrescenta que os valores de albedo e Rn observados para as áreas de pastagem, sucessão secundária e floresta ombrófila aberta na regiáo da RBJ foram similares aos verificados para as mesmas classes na regiáo da FNSA, evidenciando a boa proximidade entre as estimativas e a consequente relaçáo com os estudos apresentados anteriormente envolvendo o modelo SEBAL.

Foi constatada nas imagens correspondentes à regiáo da FNSA a presença de um ruído do tipo striping (elipses tracejadas nas Figuras 4 e 5), que se deu na banda 5. Esse tipo de ruído é comum em imagens de sensoriamento remoto e ocorre devido a falhas de detectores. Para que esse ruído não afetasse a análise do trabalho e visando manter as características iniciais dos dados orbitais utilizados, optou-se por não aplicar nenhum filtro para correção.

Observou-se nas imagens referentes à regiáo da RBJ que algumas áreas de floresta ombrófila aberta apresentaram valores de Rn destacadamente mais baixos do que outras, como pode ser visto nos círculos tracejados na Figura 7. Segundo Silva $e t$ al. (2011), a inclinação da superfície influência na incidência de radiaçáo solar e, consequentemente, no Rn. Dessa forma, como alguns pontos da regiáo da RBJ possuem um relevo mais acidentado, a incidência de radiação é menor nessas áreas, em alguma parte do dia, ocasionando assim uma diminuição no Rn. Atenta-se que esse fato não foi observado para a região da FNSA, por essa apresentar um relevo mais suave em sua totalidade. Nos últimos anos, alguns estudos (Allen et al. 2007; Di Pace et al. 2008) têm levado em consideração os efeitos topográficos na estimativa de Rn, assim sendo, poderiam ser realizados estudos em regióes de relevo mais acidentado na Amazônia, empregando modelos que utilizassem informaçóes numéricas do terreno, como por exemplo, o algoritmo METRIC (Allen et al. 2007).

A equaçáo aplicada para o cômputo do LAI subestimou os valores tanto sob as condiçōes de pastagem quanto de floresta, porém, de maneira menos acentuada na pastagem. Liberato (2011), em um trabalho em que se estimou o LAI (a partir da mesma equaçáo utilizada no presente estudo) em áreas de floresta e pastagem em RO através de dados TM/Landsat 5, também observou, na comparação com a literatura científica, valores mais consistentes para áreas de pastagem do que para áreas de floresta. Nesse sentido, salienta-se que a imprecisão no cômputo dessa variável pode ter incorrido em propagaçóes de erro nas estimativas posteriores do modelo (emissividade, radiaçáo IVT emitida e Rn). Esse fato aponta para duas necessidades importantes em estudos futuros: realizar testes de sensibilidade de modo a inferir o quão sensíveis são as estimativas posteriores do modelo ao cálculo do LAI; e calibrar a equação para estimativa do LAI por dados MODIS, ou, até 
mesmo, utilizar o produto disponibilizado pelo United States Geological Survey (USGS) (denominado MOD15A2).

Os ERs verificados para as estimativas dos fluxos de radiaçáo e albedo para o sítio de floresta $(<16 \%)$ foram semelhantes aos obtidos para o sítio de pastagem $(\leq 19 \%)$, estando de acordo com diversos estudos envolvendo o cálculo dos componentes do Rn com o modelo SEBAL, a partir de dados orbitais, apresentados anteriormente.

De acordo com os resultados obtidos com o sensor MODIS/TERRA pode-se dizer que a moderada resolução espacial desses dados pode ter influenciado no erro das estimativas, pois cada pixel pode englobar uma grande variedade de tipos de uso/cobertura da terra. No entanto, ao considerar que o erro instrumental associado à obtençăo de Rn é de 2,5\% (Gomes 2009), a obtenção de ERs de 1,7\% (sítio da RBJ) e 16,3\% (sítio da FNSA) obtidos em imagens MODIS/TERRA (nas quais um pixel corresponde a 62.500 $\mathrm{m}^{2}$ ) representa um resultado relevante e evidencia a utilidade prática destes dados para uma regiáo extensa e diversa como a Amazônia.

\section{CONCLUSÕES}

As variáveis estimadas pelo SEBAL $(\alpha, \mathrm{K} \downarrow, \mathrm{L} \downarrow, \mathrm{L} \uparrow, \mathrm{Rn}$ e $\mathrm{Rn}_{24 \mathrm{~h}}$ ) variaram de acordo com o tipo de uso/cobertura da terra e mostraram coerência com os valores encontrados por outros estudos utilizando o SEBAL em tipos de uso/cobertura da terra semelhantes.

A validação das estimativas para as condições de pastagem e floresta indicaram uma excelente à razoável concordância com as medidas in situ. Na região da FNSA os ERs variaram entre 0,2 e 19,2\%, enquanto que na regiáo da RBJ variaram de 0,8 a $15,6 \%$.

A integraçáo de dados de sensoriamento remoto (MODIS/ TERRA) com informaçóes de superfície (torres do LBA), por intermédio do modelo SEBAL, constituiu uma proposiçâo útil para a estimativa e espacialização dos componentes do balanço de radiaçáo na regiâo amazônica, o que pode contribuir para a melhor compreensão da interaçáo entre a floresta tropical e a atmosfera e gerar informaçôes de entrada necessárias aos modelos de superfície acoplados aos modelos de circulaçấo geral da atmosfera.

\section{AGRADECIMENTOS}

Os autores agradecem à Msc. Renata Gonçalves de Aguiar, do Escritório Regional do Projeto LBA de Ji-Paraná (RO), pela disponibilização dos dados micrometeorológicos; ao Dr. Celso Von Randow, do Centro de Ciência do Sistema Terrestre / INPE, pelo auxílio no tratamento das informaçóes de superfície; e à CAPES pela concessão de bolsa de mestrado ao primeiro autor.

\section{BIBLIOGRAFIA CITADA}

Aguiar, L.J.G. 2007. Balanço de radiação em áreas de floresta e pastagem em Rondônia. Dissertação de Mestrado, Universidade Federal de Viçosa, Viçosa, Minas Gerais, 70 pp.

Allen, R.G.; Tasumi, M.; Trezza, R. 2002. SEBAL (Surface Energy Balance Algorithms for Land) advanced training and user's manual - Idaho implementation. Idaho University, Idaho, USA. 98 pp.

Allen, R.G.; Tasumi, M.; Trezza, R. 2007. Satellite-based energy balance for mapping evapotranspiration with internalized calibration "METRIC" - model. Journal of Irrigation and Drainage Engineering, 133 (4): 380-394.

Artaxo, P. 2012. Break down boundaries in climate research. Nature, 481 (7381): 239-239.

Bastiaanssen, W.G.M. 1995. Regionalization of surface flux densities and moisture indicators in composite terrain. Tese de Doutorado, Wageningen Agricultural University, Wageningen. 273 pp.

Bastiaanssen, W.G.M.; Noordman, E.J.M.; Pelgrum, H.; Davids, G.; Allen, R.G. 2005. SEBAL for spatially distributed ET under actual management and growing conditions. Journal of Irrigation and Drainage Engineering, 131 (1): 85-93.

Bhattarai, N.; Dougherty, M.; Marzen, L.J.; Kalin, L. 2012. Validation of evaporation estimates from a modified surface energy balance algorithm for land (SEBAL) model in the southeastern United States. Remote Sensing Letters, 3 (6): 511-519.

Boegh, E.; Soegaard, H.; Thomsen, A. 2002. Evaluating evapotranspiration rates and surface conditions using Landsat TM to estimate atmospheric resistance and surface resistance. Remote Sensing of Environment, 79 (2-3): 329-343.

Cardoso, M.; Nobre, C.A.; Sampaio, G.; Hirota, M.; Valeriano, D.M.; Câmara Neto, G. 2009. Long-term potential for tropicalforest degradation due to deforestation and fires in the Brazilian Amazon. Biologia, 64 (3): 433-437.

Culf, A.D.; Esteves, J.L.; Marques Filho, A.O.; Rocha, H.R. 1996. Radiation, temperature and humidity over forest and pasture in Amazonia, p. 175-192. In: Gash, J.H.C.; Nobre, C.A.; Roberts, J.M.; Victoria, R.L. (Eds.). Amazonian deforestation and climate. John Wiley \& Sons, New York.

Culf, A.D.; Fisch, G.; Hodnett, M.G. 1995. The albedo of Amazonian forest and ranchland. Journal of Climate, 8 (6): 1544-1554.

Di Pace, F.T.; Silva, B.B.; Silva, V.P.R.; Silva; S.T.A. 2008. Mapeamento do saldo de radiação com imagens Landsat 5 e modelo de elevaçáo digital. Revista Brasileira de Engenharia Agricola e Ambiental, 12 (4): 385-392.

Galvão, J.A.C.; Fisch, G. 2000. Balanço de energia em áreas de floresta e de pastagem na Amazônia (Ji-Paraná, RO). Revista Brasileira de Meteorologia, 15 (2): 25-37.

Gash, J.H.C.; Huntingford, C.; Marengo, J.A.; Betts, R.A.; Cox, P.M.; Fisch, G.; Fu, R.; Gandu, A.W.; Harris, P.P.; Machado, L.A.T.; Von Randow, C.; Silva Dias, M.A.F. 2004. Amazonian climate: results and future research. Theoretical and Applied Climatology, 78 (1-3): 187-193. 
Giongo, P.R. 2011. Mapeamento do balanço de energia e evapotranspiraçáo diária por meio de técnicas de sensoriamento remoto. Tese de Doutorado, Universidade de São Paulo, Piracicaba, São Paulo. 122 pp.

Gomes, H.B. 2009. Balanços de radiação e energia em áreas de cultivo de cana-de açúcar e cerrado no Estado de Sáo Paulo mediante imagens orbitais. Tese de Doutorado, Universidade Federal de Campina Grande, Campina Grande, Paraíba. 108 pp.

Huete, A.R. 1988. A soil adjusted vegetation index (SAVI). Remote Sensing of Environment, 25 (3): 295-309.

Lasslop, G.; Reichstein, M.; Papale, D.; Richardson, A.D.; Arneth, A.; Barr, A.; Stoy, P; Wohlfahrt, G. 2010. Separation of net ecosystem exchange into assimilation and respiration using a light response curve approach: critical issues and global evaluation. Global Change Biology, 16 (1): 187-208.

Liang, S. 2001. Narrowband to broadband conversions of land surface albedo I algorithms. Remote Sensing of Environment, 76 (2): $213-238$.

Liberato, A.M. 2011. Estimativa do albedo e índice de área foliar na Amazônia. Revista Brasileira de Geografia Física, 4 (1): 22-32.

Liberato, A.M. Saldo de radiação usando imagens Landsat 5-TM na Amazônia. 2010. In: Congresso Brasileiro de Meteorologia XVI, Belém. Anais... Rio de Janeiro: CBM, 2010. p. 1-5.

Lira, V.M. 2008. Obtenção de parâmetros biofísicos e evapotranspiração da cana-de açúcar mediante imagens orbitais na regiáo do sub médio São Francisco. Tese de Doutorado, Universidade Federal de Campina Grande, Campina Grande, Paraíba. 161 pp.

Marx, A.; Kunstmann, H.; Schuttmeyer, D.; Moene, A.F. 2008. Uncertainty analysis for satellite derived sensible heat fluxes and scintillometer measurements over savannah environment and comparison to mesoscale meteorological simulation results. Agricultural and Forest Meteorology, 148 (4): 656-667.

Moraes, E.C.; Franchito, S.H.; Rao, V.B. 2003. Effects of biomass burning in Amazonia on climate: A numerical experiment with a statistical-dynamical model. Journal of Geophysical Research, 109 (D05109): 1-12.

Nobre, C.A.; Oliveira, G.S.; Velasquez, L.F.S. 2007. Mudanças climáticas e Amazônia. Ciência e Cultura, 59 (3): 22-27.

Oliveira, G. 2012. Modelagem do balanço de energia e evapotranspiração na Amazônia Brasileira com uso de imagens MODIS E ASTER. Dissertaçáo de Mestrado, Instituto Nacional de Pesquisas Espaciais, São José dos Campos, São Paulo. 227 pp.

Oliveira, G.S.; Nobre, C.A.; Costa, M.H.; Prakki, S.; Soares Filho, B.S.; Cardoso, M.F. 2007. Regional climate change over eastern Amazonia caused by pasture and soybean cropland expansion. Geophysical Research Letters, 34 (L17709): 1-7.

Paiva, C.M.; França, G.B.; Liu, W.T.H.; Rotunno Filho, O.C. 2011. A comparison of experimental energy balance components data and SEBAL model results in Dourados, Brazil. International Journal of Remote Sensing, 32 (6): 1731-1745.
Roberts, J.M.; Cabral, O.M.R.; Da Costa, J.P.; McWilliam, A.L.C.; Sá, T.D. 1996. An overview of the Leaf Area Index and physiological measurements during ABRACOS, p. 287-305. In: Gash, J.H.C.; Nobre, C.A.; Roberts, J.M.; Victoria, R.L. (Eds.). Amazonian deforestation and climate. John Wiley \& Sons, New York.

Rojas, F.; Schowengerdt, R.A.; Biggar, S.F. 2002. Early results on the characterization of the Terra MODIS spatial response. Remote Sensing of Environment, 83 (1-2): 50-61.

Ruhoff, A.L.; Paz, A.R.; Collischonn, W.; Aragão, L.E.O.C.; Rocha, H.R.; Malhi, Y.S. 2012. A MODIS-Based Energy Balance to Estimate Evapotranspiration for Clear-Sky Days in Brazilian Tropical Savannas. Remote Sensing, 4 (3): 703-725.

Santos, C.A.C.; Nascimento, R.L.; Rao, T.V.R.; Manzi, A.O. 2011 a. Net radiation estimation under pasture and forest in Rondônia, Brazil, with TM Landsat 5 images. Atmósfera, 24 (4): 435-446.

Santos, C.A.C.; Silva, B.B.; Rao, T.V.R.; Satyamurty, P.; Manzi, A.O. 2011b. Downward longwave radiation estimates for clear-sky conditions over northeast Brazil. Revista Brasileira de Meteorologia, 26 (3): 443-450.

Silva, B.B.; Braga, A.C.; Braga, C.C. 2011. Balanço de radiação no perímetro irrigado São Gonçalo - $\mathrm{PB}$ mediante imagens orbitais. Revista Caatinga, 24 (3): 145-152.

Silva, B.B.; Lopes, G.M.; Azevedo, P.V. 2005. Balanço de radiação em áreas irrigadas utilizando imagens Landsat 5 - TM. Revista Brasileira de Meteorologia, 20 (2): 243-252.

Souza, P.J.O.P.; Ribeiro, A.; Rocha, E.J.P; Loureiro, R. S.; Bispo, C.J.C; Sousa, A.M.L. 2010. Albedo da cultura da soja em área de avanço da fronteira agrícola na Amazônia. Revista Brasileira de Engenharia Agricola e Ambiental, 14 (1): 65-73.

Von Randow, C.; Manzi, A.O.; Kruijt, B.; Oliveira, P.J.; Zanchi, F.B.; Silva, R.L.; Hodnett, M.G.; Gash, J.H.C.; Elbers, J.A.; Waterloo, M.J.; Cardoso, F.L.; Kabat, P. 2004. Comparative measurements and seasonal variations in energy and carbon exchange over forest and pasture in South West Amazonia. Theoretical and Applied Climatology, 78 (1-3): 5-26.

Wang, Q.; Adiku, S.; Tenhunen, J.; Granier, A. 2005. On the relationship of NDVI with leaf area index in a deciduous forest site. Remote Sensing of Environment, 94 (2): 244-255. Wright, I.R.; Nobre, C.A.; Tomasella, J.; Rocha, H.R.; Roberts, J.M.; Vertamatti, E.; Culf, A.D.; Alvalá, R.C.S.; Hodnett, M.G.; Ubarana, V.N. 1996. Towards a GCM surface parameterization of Amazonia, p. 473-504. In: Gash, J.H.C.; Nobre, C.A.; Roberts, J.M.; Victoria, R.L. (Eds.). Amazonian deforestation and climate. John Wiley \& Sons, New York.

Zanchi, F.B.; Waterloo, M.J.; Aguiar, L.J.G.; Von Randow, C.; Kruijt, B.; Cardoso, F.L.; Manzi, A.O. 2009. Estimativa do Índice de Área Foliar (IAF) e Biomassa em pastagem no estado de Rondônia, Brasil. Acta Amazonica, 39 (2): 335-348.

Recebido em: 14/04/2012

Aceito em: 21/09/2012 
